\title{
Evaluation of Intelligent Agent Frameworks for Human Learning in Virtual Worlds
}

\author{
http://dx.doi.org/ijep.v1i3.1816 \\ M. Soliman and C. Gütl \\ Graz University of Technology, Graz, Austria
}

\begin{abstract}
Pedagogical Agents are intelligent agents supporting learning in virtual learning environments, VLE. The use of the multi-agent society model inhabited with intelligent virtual agents has shown to provide several benefits to learning. This paper reviews intelligent agents for learning and shows their educational value while demonstrating the new learning possibilities supported by them. Towards the objective of efficiently utilizing the agents in a distributed learning platform, the paper provides an evaluation of intelligent agent development frameworks. This evaluation will provide valuable information to those employing and integrating intelligent agents for different types of VLE with a view towards creating new learning scenarios.
\end{abstract}

Index Terms-Intelligent Agents, Intelligent Pedagogical Agents, Virtual Learning Environments, Virtual Worlds for Learning.

\section{INTRODUCTION}

With the explosive proliferation of Internet services and the availability of computing resources, the benefits of electronic learning have become more evident leading to new learning paradigms. Several possibilities are emerging that have recently shown acceptance to the use of 3D virtual learning environments and virtual worlds, [1]. Those environments make enormous resources available and provide new possibilities of active explorative learning and new methods of communication in 3D environments while having rich visualization effects.

Education theory and practice mandate utilizing best practices for learning to follow a discipline in understanding how learning occur in the minds of our students and develop effective learning methods based on it. Artificial Intelligence has its roots in understanding human learning functions while simulating it with several methods. That leads to different intelligent computational models. One such model is the use of multiple interacting intelligent software entities named agents that are completely different from a traditional program or object. That agent module is rather intelligent, autonomous, acts on the environment based on sensing mechanisms. This model is shown to have a distributed model of computation that suits explorative and just-in-time learning that can also support the collaborative learning in environments such as virtual worlds, [2]. Our research targets this model of intelligence investigating pedagogical values to electronic learners while attempting the evolving virtual world paradigm as a VLE as possible.

Intelligent Agents have been used as cognitive tutors utilizing cognitive models of learners and providing a scaffold for personalized learning. They have been also used as companions that can provide emotional support to learners, exemplified by embodied agents, [1]. Those agents are becoming better equipped with abilities that can provide more engagement and motivation in the electronic environment. They can be used to complement avatars for learning in virtual worlds such as second life and Open Wonderland Multi-User Virtual Environment, MUVE, [1][5]. The agent can act as a focal point for interaction between the learner and the MUVE with several patterns demonstrated.

This paper will provide motivation of intelligent agents from educational perspectives; will provide its developments in research. And therefore, provides an input to finding an answer to the question of what agent characteristics can provide a good match to our requirements by visiting different development platforms.

The paper is organized as follows. Section 2 provides insights into agent systems for human learning in virtual learning environments. Section 3 provides input to the evaluation of several available agent frameworks highlighting interesting properties. Section 4 provides a discussion and conclusion.

\section{AGENT SYSTEMS FOR HUMAN LEARNING}

In research literature, several research efforts employ intelligent agent frameworks for learning. Pedagogical gains of those can be highlighted in [3]. Our interest in this paper is to complement our prior efforts by investigating how available agent frameworks can help achieving those pedagogical functions.

The proper selection of an agent framework depends on the environment and on agent abilities such as learner knowledge representation and the ability to capture learner emotional, mental state and prior knowledge by interacting with the learner or with other agents. Forming preliminary selection criteria to find how agent methods and frameworks can help achieve our goal. Then, the different state-of-the-art agent frameworks can be inspected to find a fulfilling one to the requirements.

Intelligent agent research is relatively not new compared to the discipline of virtual worlds for human learning. It is found that there are considerable efforts in employing intelligent agents in other traditional non-learning environments and in Intelligent Tutoring Systems. Therefore, the characteristics of the new learning environment need to be considered. Agents will inhabit those environments to provide extra learning functions. Virtual Learning environments can take various forms. Recently virtual worlds have taken considerable attention from worldwide learning institutions and research as an instruction delivery mechanism. They use them to take advantage of rich visualization, multi-modal communication methods, as 
well as the gaming-like attraction. For example, several projects try to build learning environments/scenarios on top of SecondLife and Open Wonderland MUVEs.

We can look into two aspects of learning with agents; by looking at both individual agent functions interacting with the learner and at the agents' collective behaviour of an agent society interacting within the environment. Those required functionalities are inspired from either pedagogical agent research or from Intelligent Multi Agent systems, MAS.

\section{A. Overview of Agent Functions for Learning in Virtual Learning Environments}

In research literatures, intelligent agent systems have been used for human learning purposes either individually as pedagogical agents, collectively in agent societies, or within virtual learning environments. The use of those agents can provide different learning functions, [3]. Those agents can be depicted as:

a) Agents for learning personalization. Those agents promote learning through understanding individual learning abilities and treating the learner accordingly.

b) Agents for emotional support: Those agents support learning through improving engagement and motivation in the learning environment through considering the learner emotional state and improving it accordingly.

c) Cognitive Agents. Those agents are inspired from cognitive theories of the human mind as well as AI.

d) Meta-cognitive agents. Considers meta-cognition.

e) Teachable Agents. Those agents improve learning by giving the human learner the ability to teach an artificial pedagogical agent.

f) Self Regulated Learning Agents. Apply Selfregulated learning theories by agents.

g) Conceptual Change Agents. Agents that consider conceptual change learning theories.

h) Multiple agents supporting group learning or training

Considering the available agent framework, we need to look into further functional perspectives. The agentbased implementation of those functions is taken individually by different research groups. Definitely, adopting most of those functions simultaneously in the Virtual Environment is desirable with need of considering Agent Frameworks.

\section{B. Pedagogical Agents Functional Requirements}

Those requirements can be grouped into:

i) Learner interface requirements. This requirement is relevant to pedagogical agent abilities that can relate to:
a. Believability of the agent.
b. Expression and capturing of learner emotions.

c. Animation abilities to be able to navigate through a virtual world while providing expression of emotions

j) Autonomy (see [10]). Agent autonomy and selfcontrol are desirable features in 3D virtual words. Currently characters in the virtual world are more user-controlled (avatars). Robotic or autonomous avatars will give rise to learning functions in virtual worlds such as interaction and explanations of lessons, 3D scenes or objects.

k) Cognitive abilities: For the pedagogical agent to provide learning support to the learner or for the agent to resemble a human, strong cognitive abilities are required. The Belief-Desire-Intention model, BDI represents a core feature of agent systems implementing those functions. The BDI implementation comprises an important factor in selecting an agent framework.

1) Agent Social abilities: It is the ability of the agent to interact with other agents to cooperate to achieve a collective goal, negotiate and resolve a conflict. This requirement can give benefits to group learning for achieving group learning objectives. Agent societies may constitute a distributed paradigm for decision support for learning purposes. Multi-agent intelligent systems are used for decision support that can also involve team training and its relevant decision making.

m) Environment and Context Awareness: The ability of the agent to be able to navigate a virtual world discovering, constructing or suggesting learning resources, scenarios or scenes that are suitable to learner abilities and goals. Integrating the agent framework may not be straightforward.

\section{INTELLIGENT AGENT FRAMEWORKS FOR INCORPORATING DISTRIBUTED INTELLIGENCE}

We can take two approaches for deploying agent functions in a virtual learning environment; 1) developing agent functions in the VLE itself using the tool or 2) deploying an already developed framework and integrating it in the VLE. The first choice somehow implies reinventing the wheel of intelligent agents' DAI implementations. Although the second choice seems plausible, it might not be easily implementable due to the differences in implementations of the VLE and the platform design restrictions. For example, equipping the avatar in Open Wonderland versus Second Life with an intelligent agent functions requires awareness of availability of this possibility as well as the agent framework. [6] reports the difficulty of integrating an agent framework into Second Life suggesting an interface solution. Therefore, this issue is considered seeking prior project implementations lessons.

Several research and commercial agent platforms are already available and are becoming more mature across time. The functions of those platforms are not trivial to save the effort of developing agent functions from scratch. Nevertheless, investigating the potential agent platform can help us add intelligence for learning in a MUVE such as Virtual Worlds and serve as a step ahead towards our objective. Since there are numerous agent platforms found, we will focus on some of them based on function- 
alities, popularity, maturity, standardization, projects implemented, and potential integration into Virtual Worlds.

Our work considers new learning scenarios of robotic avatars moving in intelligent domains to recommend resources to learners or interacting with other non-robotic avatars for group learning supports. One possible scenario is an agent or robotic avatar that is constructing artificial scenery in a virtual world that is adapted to specific learner ability and a goal. The agent will aid the learner, interact with intelligent objects in the constructed virtual world to support learning or more. Therefore, the construction of autonomous agents in a virtual world/environment can make this feasible.

\section{A. $3 A P L$}

3APL is a tool and programming language for the development of intelligent cognitive agents based on the BDI model. It controls agent behaviour by using actions, beliefs, goals, plans, and rules. 3APL is developed and maintained in the University of Utrecht, Netherlands.

\section{B. JACK}

Jack, [8][11] is a commercial multiagent framework based on Java with development history starting in 1997. Jack is equipped with a graphical Jack Development Environment, JDE. Interoperability in Jack depends on its implementation on Java. Jack is relatively strong framework supporting BDI as reported in [4]. Agents in Jack post events which other agents can respond to by executing an agent plan (sequence of actions the agent will take responding to an event). Those events can be normal or BDI events. Jack agents possess beliefs (Belief-set class) with changes to those beliefs can trigger BDI events. An agent can have different plans to respond to depending on their relevance or context. Figure 1 shows the agent design tool in Jack with a Jack agent having two plan types to respond to an event depending on the event relevance. In Jack, capabilities are functionalities that can be 'plugged in' to the agent which gives rise to the extension abilities of the Jack framework. Based on Jack, CoJack is a BDI cognitive architecture for modeling human behavior thus allowing for humanoids/virtual actors development. The Jack Teams product extends Jack to provide a team oriented modeling framework. An example of Jack utilization is a Jack-based Pedagogical Agent in a virtual world that is executing different learning plans suitable to the learner abilities as well as the navigation context.

\section{JADE}

JADE (Java Agent Development Environment), [7] is an open source Java-based framework. JADE is popular for following the FIPA (Foundation for Intelligent Physical Agents) specifications including the FIPA-ACL, Agent Communication Language standard. JADE is comprised of a set of graphical tools for agent-based design and development. One major feature of JADE is its distribution oriented architecture that can help in performance issues. JADE has a wide range of implementations and research projects found in literature. BDI is not directly implemented in JADE but through JADEX or the BDI4JADE extension layer, [13]. Furthermore, research by [5] reported integration of JADE with Open Wonderland showing human learning benefits.

\section{AgentSpeak/Jason}

AgentSpeak is a well-known logic-based agent programming language that started in 1996 based on BDI. AgentSpeak agent creation specifies a set of beliefs, plans, and goals. A plan is triggered by an environment event. The agent can have two types of goals; test goals or achievement goals. An achievement goal is an environment state that the agent wants to reach and the test goal is relevant to belief updates. Jason is an open source Javabased interpreter extension to AgentSpeak to allow the programming of cognitive agents and the behavior of the individual agent. With Jason, further new features are added such as speech-act based communication, ontological support and belief revision abilities, [12]. However, [12] reports deficiencies in belief revisions that can be also found in other platforms thus provides a solution to the problem with Jason. Another addition to AgentSpeak/Jason is AF-AgentSpeak which is a collection of platforms, tools, and languages for agent development and deployment. With AF-AgentSpeak, relevant projects are implemented; NEXUS is a project to build virtual characters, MiRA as a Mixed Reality Agents, and AF-EISOpenSim. AF-EIS-OpenSim is a project in the University College of Dublin, UCD to integrate AF-agents with OpenSim which is the technology behind Second Life, See Figure 2, [14].

\section{E. GOAL}

GOAL is a programming language and platform for developing intelligent agents, [9]. Agent actions in GOAL are derived from beliefs and goals. While GOAL is Java based, it allows knowledge representation of goals and be-

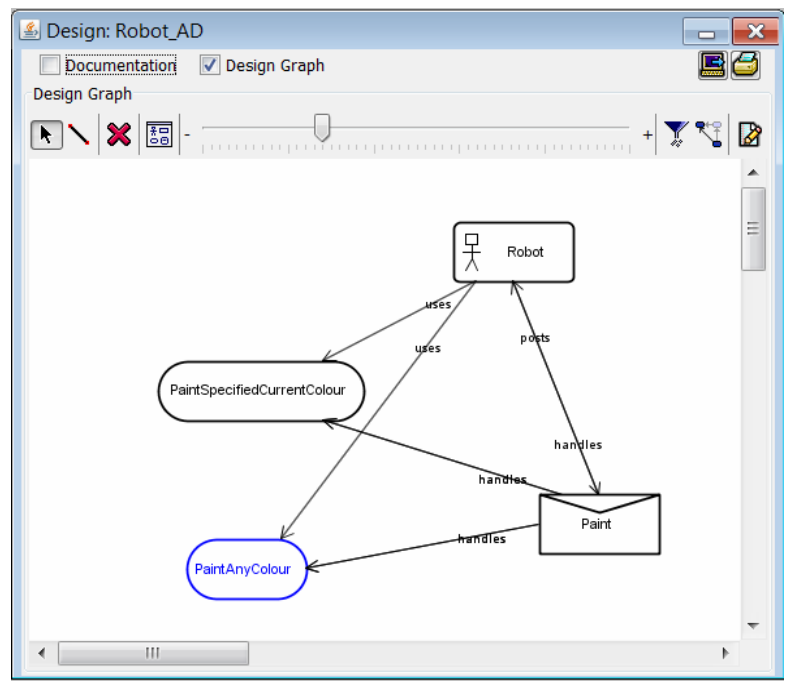

Figure 1. Graphical representation of a Jack robot agent with two plan types responding to an event, Jack practicals.

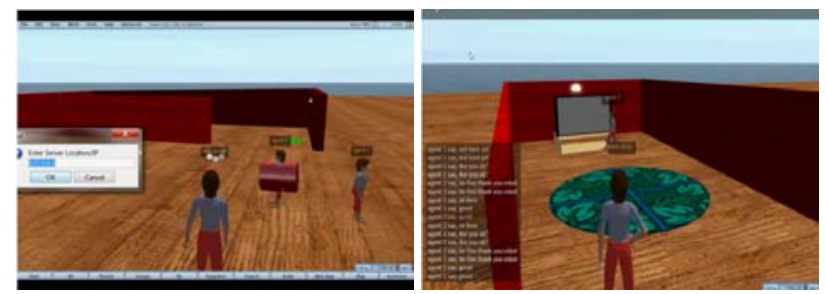

Figure 2. Snapshots from AF-agents controlling OpenSim Avatars,

[14] 
liefs with Prolog. It is reported that GOAL is advantageous to other frameworks as it offers a declarative only goal definitions method separating goal declaration from the way to achieve it.

\section{DISCUSSION AND CONCLUSION}

Several agent systems are available and are becoming more mature with varying distributed intelligent applications. Also, several software tools are available to build pedagogical agents. However, there is a gap between them especially considering MAS support and integration in virtual worlds. It is required to take advantage of MAS state-of the art and common functionalities such as Beliefs, BDI, Belief revision functions, and other cognitive abilities. Despite the complexity level of implementing pedagogical functions by agents, functions such as support of constructivism, scaffold functions, and collaborative learning need to be supported by the MAS not individually but within a rich learning environment such as virtual worlds for learning. An Example of the demanded functions is to be able to construct a pedagogical agent-based virtual learning scenario/scene based on prior learner background. This mandates coordination of several components including the user model, the learning objectives, the environment, and other learners' relevant information.

This paper investigated common tools for building agent systems, found in literature, showing common features such as BDI support, beliefs, goals, plans, java compatibility, and tools availability. In spite of the proliferation of virtual worlds and given their learning potential, several agent frameworks lack to service them. Selecting a proper agent toolkit is not a trivial task, but relies on maturity of the framework, the supporting functions, performance and the degree of integration with the targeted learning environment. The open architecture of virtual worlds mandates an agent platform that is also open to communicate and interoperate with other agent platforms. Thus selecting a standard following platform is highly recommended.

\section{REFERENCES}

[1] M. Soliman and C. Guetl, "Intelligent Pedagogical Agents in Immersive Virtual Learning Environments: A Review“ MIPRO 2010, May 2010, Opatija, Croatia.

[2] M. Soliman and C. Guetl, "Review and Perspectives on Intelligent Multi-Agent Systems’ Support for Group Learning” ED-MEDIA 2010, June 2010, Toronto, Canada.
[3] M. Soliman and C. Guetl, "A Survey of Pedagogical Functions of Intelligent Agents in Virtual Learning Environments, “Accepted for publication in the Journal of Internet Technologies, Special Issue of Agent Technology in Cyberspace. 2011.

[4] C. Cheong, "A Comparison of JACK Intelligent Agents and the Open Agent Architecture"

[5] J. Blair and F. Lin, "An Approach for Integrating 3D Virtual Worlds with Multiagent Systems., “ 2011 Workshops of International Conference on Advanced Information Networking and Applications.

[6] S. Ranathunga, S. Cranefield, and M. Purvis, "Interfacing a Cognitive Agent Platform with a Virtual World: a Case Study using Second Life, “ in Proc. of 10th Int. Conf. on Autonomous Agents and Multiagent Systems (AAMAS 2011), May, 2-6, 2011, Taipei, Taiwan, pp. 1181-1182.

[7] JADE, Java Agent DEvelopment Framework, http://jade.tilab.com/, Online, Accessed August 31, 2011.

[8] JACK, http://www.agent-software.com.au/products/jack/, Online, Accessed August 31, 2011.

[9] GOAL Agent Programming Language, https://mmi.tudelft.nl/ trac/goal/ , Online, Accessed August 31, 2011.

[10] R. Aylett and M. Luck, “Applying Artificial Intelligence to Virtual Reality: Intelligent Virtual Environments”. Applied Artificial Intelligence, 2000.

[11] M. Winikoff, "JACK intelligent agents: An industrial strength platform. In Multi-Agent Programming. Springer. 2005.

[12] N. Alechina, R. Bordini, J. Hubner, M. Jago, B. Logan, "Belief Revision for AgentSpeak Agents, “AAMAS’06, May 8-12 2006, Hakodate, Hokkaido, Japan.

[13] I. Nunes, C. Lucena, and M. Luck, "BDI4JADE: a BDI layer on top of JADE, Ninth International Workshop on Programming Multi-Agent Systems (ProMAS 2011), Taipei, Taiwan, pp. 88103, 2011.

[14] The Agent Factory, http://www.agentfactory.com , Online, Accessed August 31, 2011.

\section{AUTHORS}

M. Soliman is a Ph.D. Student with Graz University of Technology, Institute for Information Systems and Computer Media, Inffeldgasse 16c, A-8010 Graz. (e-mail: Muhamed.Soliman@gmail.com).

C. Gütl Dipl.-Ing. Dr. techn. is with Graz University of Technology, Institute for Information Systems and Computer Media, Inffeldgasse 16c, A-8010 Graz. He is also an Adjunct Research Professor with the School of Information Systems, Curtin University, G P O Box U1987, Perth, Western Australia, Australia. (e-mail: Christian.Guetl@iicm.TUGraz.at).

Submitted, September, 16, 2011. Published as resubmitted by the authors 26 September 2011. 